\title{
SYNTHESIS AND PHOTOPHYSICAL STUDIES ON 5-ETHOXYCARBONYL-4- CINNAMYL-6-METHYL-3,4-DIHYDROPYRIMIDINE-2(1H)-ONE IN VARIOUS SOLVENTS
}

Sheela N. Lad, Umesh S. Mote, Swaminath L. Bhattar, Prashant V. Anbhule, Shivajirao R. Patil and Govind B. Kolekar

Fluorescence Spectroscopy Research Laboratory, Department of Chemistry, Shivaji University, Kolhapur-416004, Maharashtra, India

(Received August 11, 2008; revised April 8, 2009)

\begin{abstract}
The 5-ethoxycarbonyl-4-cinnamyl-6-methyl-3,4-dihydropyrimidine-2(1H)-one (DHPM) was synthesized via Biginelli reaction and was characterized by NMR, IR, UV-Visible absorption and fluorescence spectroscopy. The fluorescence of the compound exhibits red shift from its absorption spectra and correlated with the solvent polarity. The quantum yield of fluorescence of the DHPM was found to vary with solvent polarity. The absorption spectrum of DHPM overlaps significantly with anthracene fluorescence spectrum. Therefore fluorescence quenching experiments were performed in 1,4-dioxane. The fluorescence of anthracene was found to be quenched and quenching is in accordance with Stern-Volmer relation. The Stern-Volmer constant $\left(\mathrm{K}_{\mathrm{Sv}}=2.52\right.$ x $\left.10^{3} \mathrm{M}^{-1}\right)$ was obtained. The quenching rate constant $\left(\mathrm{k}_{\mathrm{q}}=7.145 \times 10^{11} \mathrm{M}^{-1} \mathrm{~s}^{-1}\right)$ was calculated from the fluorescence lifetime of anthracene measured on time resolved fluorimeter (TRF) in absence of DHPM. The fluorescence quenching explained on the basis of energy transfer from anthracene to DHPM derivative. The rate constant as well as efficiency of energy transfer depends on the distance between donor and acceptor and found to be $\mathrm{r}=6.39 \mathrm{~nm}$ which indicates energy transfer.
\end{abstract}

KEY WORDS: Biginelli reaction, Fluorescence quenching, Resonance energy transfer, Quantum yield, Solvatochromic effect

\section{INTRODUCTION}

Fluorescent probe has been widely used in the field of biological and organic material science. Some dihydropyrimidine derivatives are fluorescent materials that possess many valuable photophysical properties. They have received considerable interest due to their important activities such as antibacterial, antiviral, antihypertensive, antitumor and anti-inflammatory [1]. They have numerous applications in many different biological processes such as calcium channel blockers [2], $\alpha-1-\mathrm{a}$ antagonists and neuropeptide antagonists [3]. Recently some marine alkaloids such as dihydropyrimidine-5-carboxylate have been synthesized and used as fluorescent probes. They exhibit interesting biological activities like potent HIV-gp-120-CD 4 inhibitors as well as anti-HIV agents [4].

One of the important fluorescent probe BODIPY, boron dipyrromethane was used to understand the properties such as chemical and photostability, relatively high absorption coefficients and fluorescence quantum yields [5-7]. Its photophysical properties have been widely studied by absorption, fluorescence spectra and fluorescence life time measurements [812]. Solvent polarity and the local environment have profound effects on the emission spectral properties of fluorophore molecules. The effect of solvent polarity is one origin of the Stokes shift, which is one of the observations in fluorescence. In addition to specific solventfluorophore interaction, many fluorophore can form an internal charge transfer (ICT) state [13]. Therefore the role of solvent polarity is not only to lower the energy of excited state due to general solvent effects but also to govern which state has the lowest energy.

During scrutiny of literature, we envisioned that 5-ethoxycarbonyl-4-cinnamyl-6-methyl-3,4dihydropyrimidine-2(1H)-one (DHPM) is suitable for the fluorescence studies due to its

*Corresponding author. E-mail: gbkolekar@yahoo.co.in 
interesting properties. The synthesized DHPM has been well established on the basis of various spectral techniques. In this paper we report photophysical properties of DHPM by measuring absorbance and fluorescence spectrum in different solvents. From these measurements Stokes shift and fluorescence quantum yield were determined. Using FRET (fluorescence resonance energy transfer) technique, the distance between donor and acceptor was also determined.

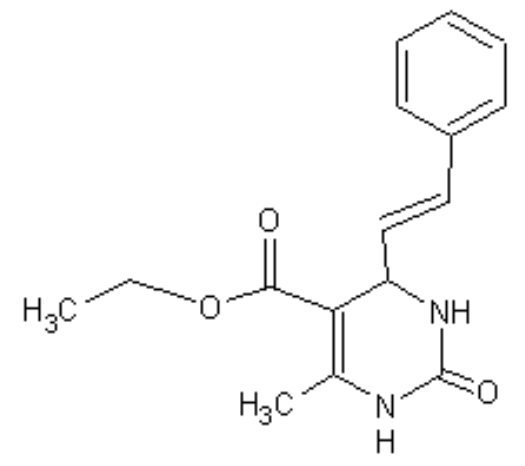

Figure 1. Molecular structure of 5-ethoxy carbonyl-4-cinnamyl-6-methyl-3,4-dihydro pyrimidine-2(1H)-one.

\section{Materials}

\section{EXPERIMENTAL}

All solvents used for synthesis and spectroscopic measurements were of analytical grade. Triple distilled water was used whenever required. Anthracene supplied by Aldrich with $99 \%$ purity was used as received. The 5-ethoxy-carbonyl-4-cinnamyl-6-methyl-3,4-dihydropyrimidine$2(1 \mathrm{H})$-one was synthesized in accordance with the method reported in literature [14] and was purified by recrystallization from ethanol. This was identified by IR, proton NMR spectral data and m.p. $230^{\circ} \mathrm{C}$. IR (KBr) $v_{\max }: 3115,3039,1710,1678,1615,1250,750 \mathrm{~cm}^{-1}$. NMR (DMSO $\left.\mathrm{d}_{6}\right) \delta, 1.5\left(\mathrm{t}, 3 \mathrm{H},-\mathrm{OCH}_{2}-\mathbf{C H}_{3}\right), 4.1$ (q, $\left.2 \mathrm{H},-\mathbf{O C H}_{2} \mathrm{CH}_{3}\right), 2.1$ (s, 3H, vinyl methyl), 5.1(1H, benzylic $\mathrm{H}), 5.8$ (d, 1H,-CH=CH-), 7.1 (m, 5H, Ar-H), 7.38 (d, 1H, $-\mathrm{CH}=\mathrm{CH}-), 8.5$ (br. s, $1 \mathrm{H}$, $\mathrm{NH}), 9.1$ (br. s, 1H, NH). C, H and N analysis found (cal.): 70.15 (70.29), H, 6.28 (6.29) and N 10.91 (10.93) and mass spectral analysis, $\mathrm{M}^{+}=256$.

\section{Spectroscopic measurements}

The UV-Visible absorption spectra were measured on Elico model SL-177 UV-Visible spectrophotometer. Fluorescence spectra were recorded on PC based Spectrofluorophotometer (JASCO Japan FP-750). Excitation and emission slit width was fixed to $10 \mathrm{~nm}$. Fluorescence life time was measured on Time Resolved Fluorescence Spectrometer (Edinburgh Analytical Instruments-FL900CDT). The IR spectrum was recorded on Perkin Elmer spectrophotometer using $\mathrm{KBr}$ pellets and NMR spectrum was obtained on Bruker $300 \mathrm{MHz}$ instrument using TMS as an internal standard. The concentration of DHPM $1 \times 10^{-3} \mathrm{M}$ was used for UV-Visible absorption and fluorescence spectra. The anthracene was used as fluorescent probe in the quenching experiments performed with DHPM quencher. Relative quantum yields $(\Phi)$ were calculated using quinine sulfate in $0.05 \mathrm{M} \mathrm{H}_{2} \mathrm{SO}_{4}$ as a reference $(\Phi=0.54)$ with the correction made for different refractive indices of solvents [15]. 


\section{RESULTS AND DISCUSSION}

\section{Absorption and fluorescence measurements}

The absorption and fluorescence spectra of DHPM $\left(1 \times 10^{-3} \mathrm{M}\right)$ were measured in different solvents at room temperature and estimated the spectroscopic data such as molar absorption coefficients, absorption and emission maxima, fluorescence quantum yields, Stokes shifts are presented in Table 1. The fluorescence emission occurs in region 385-600 nm with maximum emission at $489 \mathrm{~nm}$, some of fluorescence spectra are shown in Figure 2. The emission spectrum of the compound exhibit gradual shift from deep blue $\left(\lambda_{\max }=476 \mathrm{~nm}\right)$ in 1,4-dioxane to red in butanol $\left(\lambda_{\mathrm{em}}=489 \mathrm{~nm}\right)$. The absorption peak of DHPM in various solvents shows slight shift from $352 \mathrm{~nm}$ in THF to $358 \mathrm{~nm}$ in formamide, which indicates the large dipole moment in the excited state is higher than in the ground state because of internal charge transfer.

Table 1. Spectroscopic data of DHPM in various solvents.

\begin{tabular}{|l|c|c|c|c|c|c|}
\hline Solvent & $\begin{array}{c}\text { Solvent polarity } \\
(\Delta \mathrm{F})\end{array}$ & $\begin{array}{c}\lambda_{\mathrm{abs}} \\
(\mathrm{nm})\end{array}$ & $\begin{array}{c}\lambda_{\mathrm{em}} \\
(\mathrm{nm})\end{array}$ & $\begin{array}{c}\varepsilon \\
\left(\mathrm{M}^{-1} \mathrm{~cm}^{-1}\right)\end{array}$ & $\begin{array}{c}\text { Stoke's shift } \\
\left(\Delta v^{-} \mathrm{cm}^{-1}\right)\end{array}$ & $\begin{array}{c}\text { Quantum yield } \\
(\Phi)\end{array}$ \\
\hline 1,4-Dioxane & 0.0211 & 356 & 430 & $1.034 \times 10^{3}$ & 7081.48 & 0.0508 \\
\hline Chloroform & 0.1498 & 356 & 484 & $2.130 \times 10^{3}$ & 7428.73 & 0.0127 \\
\hline THF & 0.2243 & 352 & 481 & $2.393 \times 10^{3}$ & 7619.07 & 0.0144 \\
\hline Butanol & 0.2641 & 354 & 489 & $3.059 \times 10^{3}$ & 7798.68 & 0.0071 \\
\hline Formamide & 0.2756 & 358 & 486 & $2.232 \times 10^{3}$ & 7356.82 & 0.0150 \\
\hline Propanol & 0.2775 & 354 & 488 & $2.037 \times 10^{3}$ & 7756.78 & 0.0100 \\
\hline 2-Methyl propanol & 0.2857 & 354 & 489 & $2.151 \times 10^{3}$ & 7798.68 & 0.0117 \\
\hline Ethanol & 0.2890 & 356 & 488 & $2.260 \times 10^{3}$ & 7598.08 & 0.0108 \\
\hline Acetonitrile & 0.3056 & 352 & 488 & $2.061 \times 10^{3}$ & 7917.28 & 0.0112 \\
\hline
\end{tabular}

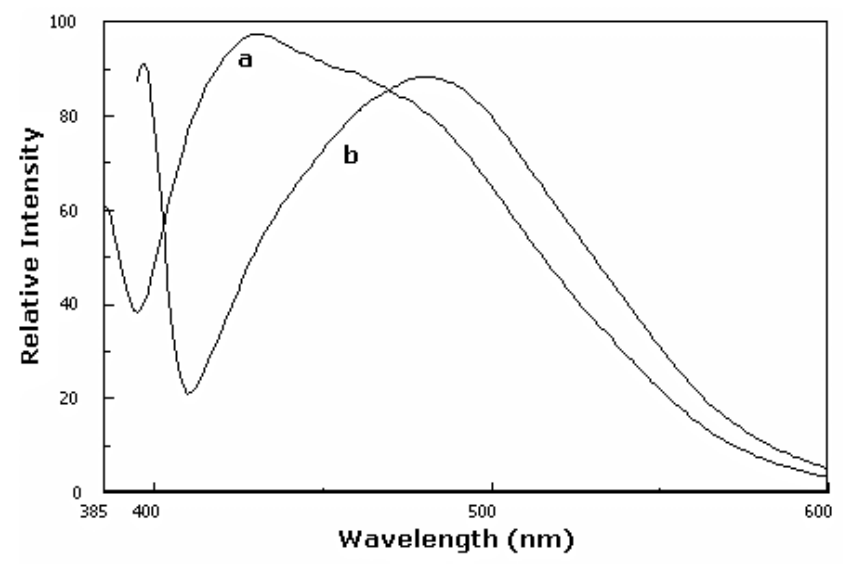

Figure 2. Fluorescence spectra of DHPM. (a) $1 \times 10^{-3} \mathrm{M}$ in 1,4-dioxane monitored at $383 \mathrm{~nm}$ excitation wavelength. (b) $1 \times 10^{-3} \mathrm{M}$ in THF at $394 \mathrm{~nm}$ excitation.

The fluorescence quantum yields $(\Phi)$ and Stokes shift $(\Delta v)$ were determined by using the following equation, 


$$
\Phi=\frac{\Phi_{s t d} \cdot F A_{s t d} n^{2}}{F_{s t d} A n_{s t d}^{2}}
$$

where $\mathrm{F}$ and $\mathrm{F}_{\text {std. }}=$ peak areas of sample and standard solutions, respectively; $\mathrm{A}$ and $\mathrm{A}_{\text {std. }}=$ absorbance at excitation wavelengths of sample and standard solutions, respectively; $n$ and $n_{\text {std. }}=$ refractive index of sample and standard solutions, respectively; $\Phi$ and $\Phi_{\text {std. }}=$ quantum yields of sample and standard solutions, respectively.

The fluorescence quantum yield of the DHPM showed slight variation with the change in solvent polarity indicating the negative and positive solvatokinetic effect. For negative solvatokinetic effect, quantum yield increases with a suitable enhancement of intramolecular charge transfer (ICT) which involves $n \pi$ electron configuration while reduction in quantum yield by strong ICT is called positive solvatokinetic effect. As can be seen from Table 1 that the highest fluorescence quantum yield was observed for 1,4-dioxan and lower for butanol solvent. The interaction between the solvent and fluorophore affect the energy difference between the ground and excited state. To a first approximation this energy difference is a property of refractive index (n) and dielectric constant $(\mathcal{C})$ of the solvent which is usually explained by the Lippert-Mataga equation [16].

$$
\begin{gathered}
\Delta v=v_{a b s}^{-}-v_{e m}^{-}=\frac{2\left(\mu_{e}-\mu_{g}\right)^{2}}{4 \pi \varepsilon_{0} h c a^{3}} \Delta f(\varepsilon, n)+c o n s \tan t \\
\Delta f=\frac{(\varepsilon-1)}{(2 \varepsilon+1)}-\frac{\left(n^{2}-1\right)}{\left(2 n^{2}+1\right)}
\end{gathered}
$$

where $\Delta v=$ Stokes shift in $\mathrm{cm}^{-1}, \mathrm{~h}=6.6262 \times 10^{-34} \mathrm{~J}$ is Planck's constant, $\mathrm{c}=2.99 \times 10^{8} \mathrm{~m} \cdot \mathrm{s}^{-1}$ is the velocity of light, and $\varepsilon_{\mathrm{o}}=$ the permittivity of vacuum $\left(8.8542 \times 10^{-12} \mathrm{C}^{2} \cdot \mathrm{N}^{-1} \cdot \mathrm{m}^{-2}\right)$.

The sensitivity of these fluorophore to solvent polarity is due to a charge shift. The solvent sensitivity of fluorophore has been estimated by a Lippert-Mataga plot. The plot of Stokes shifts versus the polarity function $\Delta \mathrm{f}$ (Figure 3 ) shows that the good correlation coefficient of Stokes shifts with solvent polarity. This result indicates that the DHPM has an ICT characters in the excited state and also the dielectric solute-solvent interactions are responsible for the observed solvatochromic shift for the present molecule.

\section{Fluorescence quenching studies}

Figure 4 shows the fluorescence quenching of anthracene (D) with the addition of DHPM (A). From the figure, it is observed that the fluorescence intensity of the donor (anthracene) decreased regularly with increasing concentration of DHPM, indicating non-radiative energy transfer between the excited donor and the accepter. The kinetics of quenching of anthracene was studied by using Stern-Volmer relation. The plot of $\mathrm{F}_{0} / \mathrm{F}$ versus concentration of quencher is shown in Figure 5, where $\mathrm{F}$ and $\mathrm{F}_{0}$ are the fluorescence intensities in presence and absence of DHPM, respectively. The Stern-Volmer plot is straight line with intercept having value one on $\mathrm{Y}$-axis and indicates validity of Stern-Volmer equation given below [17-18].

$$
\mathrm{F}_{0} / \mathrm{F}=1+\mathrm{k}_{\mathrm{q}} \tau[\mathrm{Q}]=1+\mathrm{K}_{\mathrm{sv}}[\mathrm{Q}]
$$

From the linear portion of the curve, the value of quenching rate constant $\mathrm{k}_{\mathrm{q}}$ obtained by following relation

$$
\mathrm{k}_{\mathrm{q}}=\mathrm{K}_{\mathrm{SV}} / \tau
$$


where, $\mathrm{K}_{\mathrm{SV}}$ is the Stern-Volmer constant obtained from slope, i.e. $2.52 \times 10^{3} \mathrm{M}^{-1}$ and the value of $\tau=3.53 \mathrm{~ns}\left(1 \mathrm{~ns}=1 \times 10^{-9} \mathrm{~s}\right)$ was measured on time resolved fluorimeter. The estimated value of $\mathrm{k}_{\mathrm{q}}$ was found to be $7.145 \times 10^{11} \mathrm{M}^{-1} \mathrm{~s}^{-1}$.

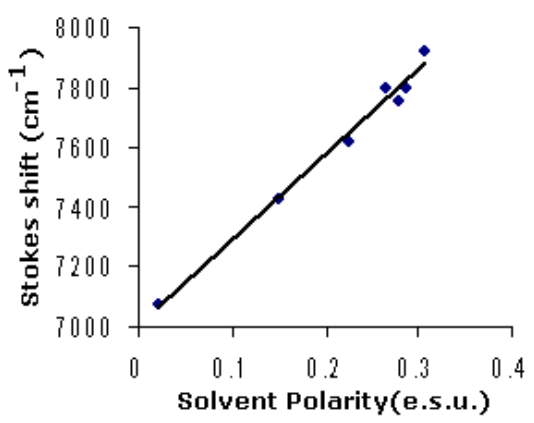

Figure 3. A plot of Stokes shift vs solvent polarity.

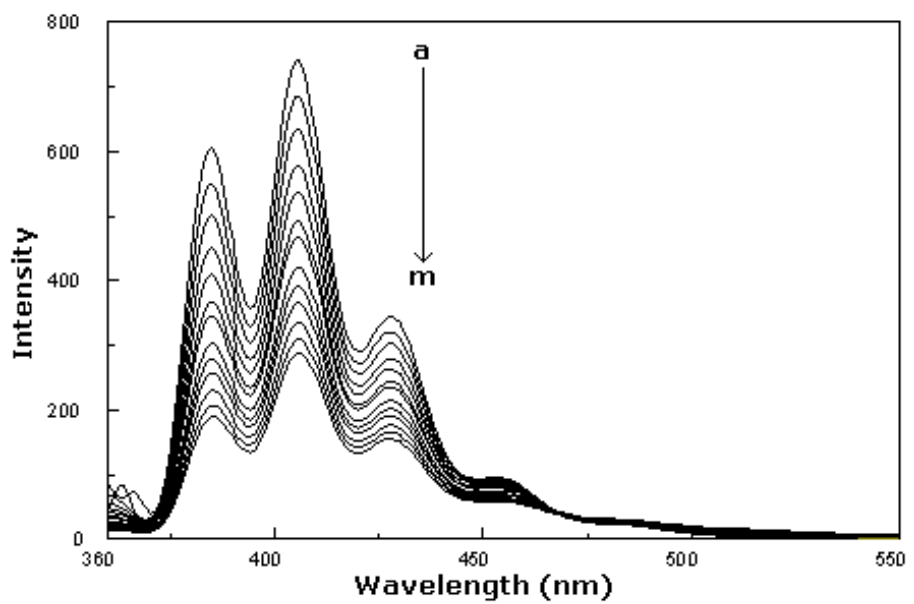

Figure 4. Fluorescence quenching of $1 \times 10^{-6} \mathrm{M}$ anthracene with varying concentrations of DHPM from a to $\mathrm{m}$ : 0.0 to $3.0 \times 10^{-4} \mathrm{M}$.

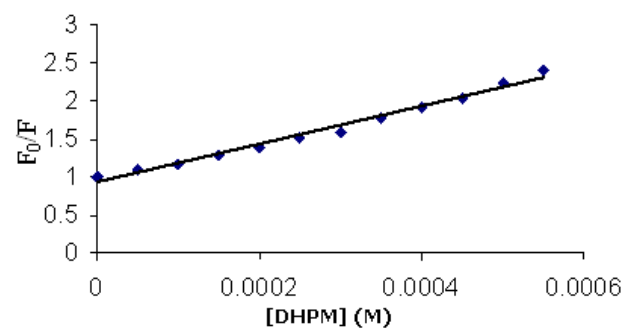

Figure 5. Stern-Volmer plot between anthracene and DHPM in 1,4-dioxane.

Bull. Chem. Soc. Ethiop. 2009, 23(2) 
Energy transfer between DHPM and anthracene

FRET is the distance-dependent transfer of energy from a donor molecule to an accepter molecule. According to Forster's theory, there are many factors that influence resonance energy transfer. The primary conditions that need to be met in order to occur FRET are relatively few. (a) The donor and accepter molecules must be in close proximity to one another (typically 10$100 \AA$ ). (b) The absorption or excitation spectrum of the accepter must overlap with the fluorescence emission spectrum of the donor. (c) The donor and accepter transition dipole orientations must be approximately parallel. These conditions have been optimized as shown in the Figure 6. Using Forster non-radiative energy transfer efficiency, E depends not only on distance $(r)$ between $\mathrm{D}$ and $\mathrm{A}$ but also on the critical energy transfer distance $\left(\mathrm{R}_{0}\right)$ expressed by the following relation [15].

$$
E=1-\left(F / F_{0}\right)=\frac{R_{0}^{6}}{R_{0}^{6}+r^{6}}
$$

where, $\mathrm{F}$ and $\mathrm{F}_{0}$ are the fluorescence intensities of anthracene in presence and absence of DHPM, $\mathrm{R}_{0}$ is the critical distance, which is estimated from following equation

$$
R_{0}^{6}=8.8 \times 10^{-25} \times K^{2} \times N^{-4} \times \Phi \times J
$$

where $\mathrm{K}^{2}=2 / 3=$ the factor expressing the spatial orientation of dipole, $\mathrm{N}=$ refractive index of medium (donor), $\Phi=$ the fluorescence quantum yield of the donor, $\mathrm{J}=$ the overlap integral of fluorescence emission spectrum of the donor and the absorption spectrum of the acceptor is given by,

$$
J=\int \frac{f(v) E(v) d v}{v^{4}}
$$

where $F(v)$ is the fluorescence intensity of donor at wavelength $\lambda, E(v)$ is the molar absorption coefficient of acceptor at wavelength $\lambda$.

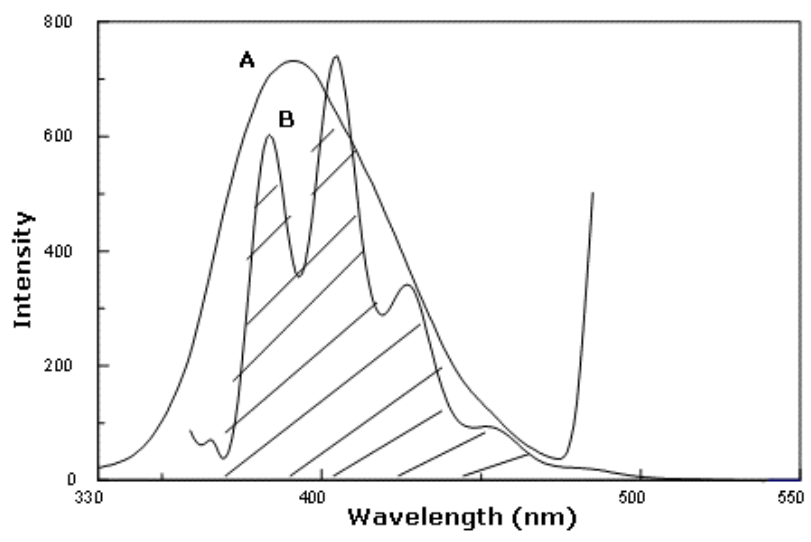

Figure 6. Spectral overlap between emission spectrum of anthracene (B) and excitation spectrum of DHPM (A). 
From the overlapping of absorption spectra of acceptor and the fluorescence spectra of donor, $\mathrm{J}=6.009 \times 10^{-14} \mathrm{~cm}^{3} \mathrm{M}^{-1}$ can be determined by integrating the spectra. In the present case $\mathrm{K}=2 / 3, \mathrm{~N}=1.4203, \Phi=0.4102$ according to equation (5-6), we could calculate the $\mathrm{R}_{\mathrm{o}}=5.23$ $\mathrm{nm}, \mathrm{E}=0.23$ and $\mathrm{r}=6.39 \mathrm{~nm}$. For Anthracene-DHPM system, the average distance, $\mathrm{r}<8 \mathrm{~nm}$ which indicates that non-radiative energy transfer from anthracene to DHPM occurs with high probability [19].

\section{CONCLUSIONS}

The titled compound DHPM was synthesized and characterized by NMR, IR spectral data. The photophysical properties of DHPM were investigated by UV-Visible, fluorescence spectra and fluorescence life time measurements in various solvents. The quantum yield of the compound differs slightly with solvent polarity. The fluorescence of anthracene was found to be quenched and quenching is in accordance with Stern-Volmer relation. The Stern-Volmer constant $\left(\mathrm{K}_{\mathrm{SV}}\right)$ $2.52 \times 10^{3} \mathrm{M}^{-1}$, quenching rate constant $\left(\mathrm{k}_{\mathrm{q}}=7.145 \times 10^{11} \mathrm{M}^{-1} \mathrm{~s}^{-1}\right)$ and the distance between anthracene (donor) and DHPM (acceptor) were calculated using FRET and found to be $r=6.39$ $\mathrm{nm}$ which indicates the effective energy transfer.

\section{AKNOWLEDGEMENTS}

Authors thank to BARC, Mumbai for providing the facility of Time Resolved Fluorimeter. We are grateful to DST and UGC for providing funds to our department under FIST and SAP programme. One of the authors (USM) acknowledges receiving a project fellowship from UGC, New Delhi [Project F.No.32-263/2006 (SR)].

\section{REFERENCES}

1. (a) Kato, T. Japn. Kokai Tokkyo Koho 1984 JP, 59; 1984, 190, 974; Chem. Abstr. 1985, 102, 132067. (b) Atwal, K.S.; Swanson, B.N.; Unger, S.E.; Floyd, D.M.; Moreland, S.; Hedberg, A.; O'Reilly, B.C. J. Med. Chem. 1991, 34, 806.

2. Atwal, K.S.; Rovnyak, G.C.; Kimball, S.D.; Floyd, D.M.; Moreland, S.; Swanson, B.N.; Gougoutas, J.Z.; Schwartz, J.; Smillie, K.M.; Malley, M.F. J. Med. Chem. 1990, 33, 2629.

3. (a) Rama Rao, A.V.; Gurjar, M.K; Vasudevan, J. J. Chem. Soc. Chem. Commun. 1995, 1369. (b) Snider, B.B.; Chen, J.; Patil, A.D.; Freyer, A. Tetrahedron Lett. 1996, 37, 6977.

4. (a) Gangadasu, B.; Palaniappan, S.; Rao, V.J. Synlett 2004, 7, 1285. (b) Yadav, J.S.; Subba Reddy, B.V.; Sridhar, P.; Reddy, J.S.; Nagaiah, K.; Lingaiah, N.; Saiprasad. P.S. Eur. J. Org. Chem. 2004, 3, 552.

5. Johnson, I.D.; Kang, H.C.; Haugland, R.P. Anal. Biochem. 1991, 198, 228.

6. Karolin, J.; Johansson, L.B.A.; Standberg, L.; Ny, T. J. Am. Chem. Soc. 1994, 116, 7801.

7. Gabe, Y.; Urano, Y.; Kikuchi, K.; Kojima, H.; Nagano, T. J. Am. Chem. Soc. 2004, 126, 3357.

8. Kollmannsberger, M.; Rurack, K.; Resch-Genger, U.; Daub, J. J. Phys. Chem. A, 1998, 102, 10211.

9. Lopez Arbeloa, T.; Lopez Arbeloa, F.; Lopez Arbeloa, I.; Garcia-Moreno, I.; Costela, A.; Satre, R.; Amat-Guerri, F. Chem. Phys. Lett. 1999, 299, 315.

10. Qin, W.; Baruah, M.; Vander Auweraer, M.; De Schryver, F.C.; Boens, N. J. Phys. Chem. A 2005, 109, 7371.

11. Qin, W.; Rohand, T.; Baruah, M.; Stefan, A.; Auweraer, M.V.; Dehaen W.; Boens, N. Chem. Phys. Lett. 2006, 420, 562. 
12. Morii, T.; Sugimoto, K.I.; Makino, K.; Otsuka, M.; Imoto, K.; Mori, Y. J. Am. Chem. Soc. 2002, 124, 1138.

13. Retting, W. Angew Chem., Int. Ed. 1986, 25, 971.

14. Deshmukh, M.B.; Anbhule, P.V.; Jadhav, S.D.; Mali, A.R.; Jagtap, S.S.; Deshmukh, S.A. Ind. J. Chem. 2007, 46B, 1545.

15. Lakowicz, J.R. Principles of Fluorescence Spectroscopy, 3rd ed., New York; 2006; p 208.

16. Ito, F.; Nagai, T.; Ono, Y.; Yamaguchi, K.; Furuta, H.; Nagamnra, T. Chem. Phys. Lett. 2007, 435, 283.

17. Azim, S.A.; Ghazy, R.; Shaheen, M.; Mekawey, F. Eur. J. Photochem. Photobiol. A 2000, $133,185$.

18. De, S.; Girigoswami, A. J. Colloid Interface Sci. 2004, 271, 485.

19. Gui, F.L.; Fan, J.; Li, J.P.; Hu, Z. Bioorg. Med. Chem. 2004, 12, 151. 